\title{
Frequency Regulation with Thermostatically Controlled Loads: Aggregation of Dynamics and Synchronization
}

\author{
Andreas Kasis and Ioannis Lestas
}

\begin{abstract}
Thermostatically controlled loads (TCLs) can provide ancillary services to the power network by aiding existing frequency control mechanisms. TCLs are, however, characterized by an intrinsic limit cycle behavior which raises the risk that these could synchronize when coupled with the frequency dynamics of the power grid, i.e. simultaneously switch, inducing persistent and possibly catastrophic power oscillations. To address this problem, schemes with a randomized response time in their control policy have been proposed in the literature. However, such schemes introduce delays in the response of TCLs to frequency feedback that may limit their ability to provide fast support at urgencies. In this paper, we present a deterministic control mechanism for TCLs such that those switch when prescribed frequency thresholds are exceeded in order to provide ancillary services to the power network. For the considered scheme, we provide analytic conditions which ensure that synchronization is avoided. In particular, we show that as the number of loads tends to infinity, there exist arbitrarily long time intervals where the frequency deviations are arbitrarily small. Our analytical results are verified with simulations on the Northeast Power Coordinating Council (NPCC) 140-bus system, which demonstrate that the proposed scheme offers improved frequency response compared with existing implementations.
\end{abstract}

\section{INTRODUCTION}

Motivation and literature review: A significant growth in the penetration of renewable sources of generation in power networks is expected over the following years [2], [3], driven by environmental concerns. This will result in increasingly intermittent generation, endangering power quality and potentially the stability of the power network. Controllable loads are considered to be a way to counterbalance intermittent generation, due to their ability to provide a fast response at urgencies by accordingly adapting their demand. The use of loads as ancillary services, in conjunction with a large penetration of renewable sources of generation will significantly increase the number of active devices in the network making its electromechanical response difficult to predict and encouraging the analytical study of its behavior. Along these lines, various

Andreas Kasis is with the KIOS Research and Innovation Center of Excellence and the Department of Electrical and Computer Engineering, University of Cyprus, Cyprus; e-mail: kasis.andreas@ucy.ac.cy

Ioannis Lestas is with the Department of Engineering, University of Cambridge, Cambridge, United Kingdom; e-mail: icl20@cam.ac.uk

This work was supported by ERC starting grant No. 679774 and by the European Union's Horizon 2020 research and innovation program under grant agreement No. 891101 (SmarTher Grid).

A preliminary version of this work has appeared in [1]. This manuscript extends the analysis to a broad class of linear generation dynamics and includes the analytic proofs of the main results, additional discussion and simulations that demonstrate the impact of the proposed analysis. research studies in recent years have considered controllable demand as a means of providing support to primary [4]-[8], and secondary [9], [10], [11], frequency control mechanisms, where the objective is to ensure that generation and demand are balanced and that the frequency converges to its nominal value $(50 \mathrm{~Hz}$ or $60 \mathrm{~Hz})$ respectively.

Thermostatically controlled loads (TCLs) comprise a significant portion of the total demand. A recent survey in the EU [12] showed that TCLs exceeded $80 \%$ and $40 \%$ of the total consumption in households with and without electric heating respectively. TCLs have an intrinsic limit cycle behavior whereby they need to periodically turn on and off in order to maintain the temperature within a prescribed range. This significantly complicates their use for frequency control, in comparison with loads that are not thermostatically controlled [11], [13], [14]. In particular, the coupling of the individual limit cycles in TCLs with the grid frequency, could lead to a synchronization of these limit cycles thus resulting to highly undesirable oscillations in the aggregate load profile. Therefore dedicated analysis tools and studies are needed for the efficient integration of TCLs to the grid such that they provide support to frequency regulation.

The use of TCLs for frequency control has been considered in [15], where the authors suggested temperature thresholds in TCLs to be linearly dependent on frequency and demonstrated with simulations that this resulted in improved performance. However, it was demonstrated in [16] that such control schemes could potentially result to load synchronization. As a remedy to this problem, the authors proposed a randomized control scheme which ensured that TCLs would not synchronize. Various other studies considered similar problems by proposing schemes with randomization in the control policy. In [17], safety constraints in the operation of TCLs are additionally included, and [18], [19] incorporate stochastic switching in the TCL operation so as to achieve a prescribed power profile. However, schemes with a randomized delay in their control policies may limit the ability of TCLs to respond to unforeseen frequency fluctuations and provide ancillary support at fast timescales. The latter, motivates the study of alternative schemes for the control of thermostatic loads, such that a faster response can be achieved at urgencies, while at the same time avoiding load synchronization.

Contribution: This study considers a deterministic approach for the control of thermostatic loads, such that ancillary services with a fast response are provided at urgencies. Our main analytic results concern the case where the number of 
loads tends to infinity, a condition justified by the large number of thermostatic appliances in power networks.

More precisely, we propose a control scheme for TCLs, such that loads switch when certain frequency thresholds are exceeded in order to support existing secondary frequency control schemes. For the considered scheme, we provide design conditions for the frequency thresholds that bound the coupling between the frequency and the load dynamics so as to avoid load synchronization. In particular, one of the main results is to analytically show that when the number of loads tends to infinity, the frequency deviations will be arbitrarily small for arbitrarily long time intervals.

The proposed scheme also ensures that load temperatures will not exceed their respective bounds, and hence that user comfort levels will not be affected. Furthermore, the fact that loads switch instantly at urgencies, leads to a fast response whereby randomized delays, often used in the literature to avoid synchronization, are avoided.

Our analytical results are verified with numerical simulations on the NPCC 140-bus network, where it is demonstrated that the proposed scheme offers reduced frequency overshoots in comparison with existing implementations.

Paper structure: In Section III we present some basic notation used in the paper and in Section [III the considered power system. In Section IV we consider a conventional model for TCLs and study its properties in terms of the aggregate mean and variance. In Section $\mathrm{V}$ we present our proposed scheme for frequency control using TCLs and state our main results regarding the performance of the power system. Numerical investigations of the results on the NPCC 140-bus system are provided in Section VI and conclusions are drawn in Section VII The proofs of the main results are provided in the appendix.

\section{NOTATION}

Real, natural and complex numbers are denoted by $\mathbb{R}, \mathbb{N}$ and $\mathbb{C}$ respectively, and the set of $n$-dimensional vectors with real entries is denoted by $\mathbb{R}^{n}$. Furthermore, we define the sets of integers and strictly positive rational and strictly positive real numbers by $\mathbb{Z}, \mathbb{Q}_{+}$and $\mathbb{R}_{+}$respectively. The set of natural numbers including zero is denoted by $\mathbb{N}_{0}$. The cardinality of a set $S$ is denoted by $|S|$. For $a \in \mathbb{R}, b \in \mathbb{R} \backslash\{0\}$, $a$ modulo $b$ is denoted by $[a]_{b}^{+}$and defined as $[a]_{b}^{+}=a-b\left\lfloor\frac{a}{b}\right\rfloor$, where for $x \in \mathbb{R}, \mid x\rfloor=\sup \{m \in \mathbb{Z}: m \leq x\}$. The average of a real valued time signal $x(t)$ with respect to time is defined as $\mathbb{E}(x(t))=\lim _{\tau \rightarrow \infty} \frac{1}{\tau} \int_{0}^{\tau} x(t) d t$ and its variance as $\mathbb{V}(x(t))=\mathbb{E}\left((x(t))^{2}\right)-[\mathbb{E}(x(t))]^{2}$. For $c \in \mathbb{C}$ we denote its magnitude by $|c|$. The 1-norm of a linear system with transfer function $G(s)$ is given by $\int_{0}^{\infty}|g(t)| d t$, where $g(t)$ is the inverse Laplace transformation of $G(s)$. We use $\mathbf{0}_{n}$ to denote the $n \times 1$ vector with all elements equal to 0 . We also say that a matrix $A \in \mathbb{R}^{n \times n}$ is Hurwitz if all its eigenvalues have strictly negative real part. Finally, a sequence $\left\{s_{1}, s_{2}, s_{3}, \ldots\right\}$ of real numbers is said to be uniformly distributed on an interval $[a, b]$ if for any subinterval $[c, d]$ of $[a, b]$ we have $\lim _{n \rightarrow \infty} \frac{\left|\left\{s_{1}, s_{2}, s_{3}, \ldots\right\} \cap[c, d]\right|}{n}=\frac{d-c}{b-a}$.

\section{POWER SYSTEM MODEL}

We use the swing equation to describe the rate of change of the frequency of the power system (e.g. [20]). In particular, we consider the following assumptions on our studied model: 1) Bus voltage magnitudes satisfy $|V|=1$ p.u. for all buses. 2) Lines are lossless and characterized by their susceptances. 3) Reactive power flows do not affect bus voltage phase angles and frequencies.

4) Frequencies between buses are synchronized.

The first three conditions have been widely used in the literature for the study of frequency control schemes in power networks [5], [11]. The fourth assumption is justified from the relatively small deviations between bus frequencies, which allows the study of power system characteristics using a single frequency (see also [16], [21], [22]). The latter follows from the fact that the dynamic behavior of TCLs is much slower than the frequency dynamics between buses, which justifies the assumption of small deviations among bus frequencies. Please note that a full complexity power network model, which includes multiple buses, voltage dynamics, line resistances and reactive power flows, is considered in the simulations presented in Section VI, which verify the main results of the paper. The above motivate the following system dynamics,

$$
M \dot{\omega}=-p^{L}+p^{M}-D \omega-\sum_{j \in N} d_{j}^{c} .
$$

In system (1) the time-dependent variables $p^{M}, d_{j}^{c}$ and $\omega$ represent, respectively, the aggregate mechanical power injection, the $j$ th thermostatic load and the deviation from the nominal value 1 of the frequency. Furthermore, we let $N:=\{1,2 \ldots,|N|\}$ be the set of TCLs. The constants $M>0$ and $D>0$ denote the generator inertia and damping coefficient respectively. Finally, the aggregate uncontrollable demand is denoted by $p^{L}$.

\section{A. Generation Dynamics}

We consider a broad class of linear generation dynamics of the form

$$
p^{M}=\hat{C} \hat{x}+\hat{D} \omega, \quad \dot{\hat{x}}=\hat{A} \hat{x}+\hat{B} \omega
$$

with input $\omega$, output $p^{M}$, state $\hat{x}$ that takes values in $\mathbb{R}^{n}$ and corresponding matrices $\hat{A} \in \mathbb{R}^{n \times n}, \hat{B} \in \mathbb{R}^{n}, \hat{C} \in \mathbb{R}^{1 \times n}$ and $\hat{D} \in \mathbb{R}$. Note that linear systems are widely used in the literature to model generation dynamics (see e.g. [20, Section 11.1], [23, Section 11.1.7]). Such models are particularly relevant when small disturbances are considered.

The system (1), (2) can be represented in the form

$$
\left[\begin{array}{c}
\dot{\omega} \\
\dot{\hat{x}}
\end{array}\right]=A\left[\begin{array}{c}
\omega \\
\hat{x}
\end{array}\right]+B\left[p^{L}+\sum_{j \in \mathcal{N}} d_{j}^{c}\right]
$$

where $A=\left[\begin{array}{cc}(\hat{D}-D) / M & \hat{C} / M \\ \hat{B} & \hat{A}\end{array}\right]$ and $B=\left[\begin{array}{c}-1 / M \\ \mathbf{0}_{n}\end{array}\right]$. We also denote $\hat{u}=p^{L}+\sum_{j \in \mathcal{N}} d_{j}^{c}$. The following assumption is made for (3).

\footnotetext{
${ }^{1}$ The nominal value is $50 \mathrm{~Hz}$ or $60 \mathrm{~Hz}$
} 
Assumption 1: For system (3) the following hold

(i) $A$ is Hurwitz,

(ii) All equilibria of (3) with constant $\hat{u}$ satisfy $\omega^{*}=0$.

Assumption 1(i) ensures that (3) is an asymptotically stable system. The latter is in line with current implementations where generation dynamics are designed such that the power system is stable. Assumption 1(ii) is associated with the fact that secondary frequency control is implemented, where the objective is to recover the frequency to its nominal value at steady state.

\section{THERMOSTATICALLY CONTROLLED LOADS}

In this section we consider a conventional model for cooling TCLs (e.g. refrigerators, air conditioner units) and study its properties. Note that the extension to heating TCLs, such as space heaters, is trivial and thus omitted. The analysis below enables to deduce important properties of TCL behavior, which are used to obtain the main results of this paper. TCL dynamics are commonly described by (e.g [16], [24])

$$
d_{j}^{c}=\bar{d}_{j} \sigma_{j}, \quad \sigma_{j}\left(t^{+}\right)= \begin{cases}1, & T_{j} \geq \bar{T}_{j}, \\ 0, & T_{j} \leq \underline{T}_{j}, \\ \sigma_{j}(t), & \underline{T}_{j} \leq T_{j} \leq \bar{T}_{j}\end{cases}
$$

where $j \in N$ and $t^{+}=\lim _{\epsilon \rightarrow 0}(t+\epsilon)$. In (4), the timedependent variables $d_{j}^{c}$, and $\sigma_{j} \in\{0,1\}$ denote the demand and switch state of the $j$ th load respectively. The time dependent variable $T_{j}$ denotes the temperature of the $j$ th load. The constants $\bar{d}_{j}, \underline{T}_{j}$ and $\bar{T}_{j}$ denote the load magnitude and lower and upper temperature thresholds for load $j$ respectively and satisfy $\bar{d}_{j} \in \mathbb{R}_{+}$and $\bar{T}_{j}>\underline{T}_{j}>0, j \in N$. The hysteresis scheme in (4) is depicted in Figure 1

Furthermore, the temperature dynamics satisfy

$$
\dot{T}_{j}=-k_{j}\left(T_{j}-\hat{T}_{j}+\lambda_{j} d_{j}^{c}\right), j \in N,
$$

where constants $k_{j}, \lambda_{j}>0$ denote the thermal insulation coefficient and coefficient of performance of load $j$ respectively. Furthermore, $\hat{T}_{j}$ denotes the ambient temperature of load $j$ that is assumed to be constant. Moreover, it is assumed that $\hat{T}_{j}-\lambda_{j} \bar{d}_{j}<\underline{T}_{j}$ and $\hat{T}_{j}>\bar{T}_{j}, j \in N$, such that (4), (5), has no equilibria, as is the case in practice.

\section{A. Periods and duty cycles of TCLs}

The period $\pi_{j}$ of thermal load $j$, described by (4), (5), is defined as the time required for load $j$ to switch twice, i.e. the time between two consecutive switches to the ON (or equivalently $\mathrm{OFF}$ ) state. In the following definition, we let $t_{j, i}$ be the time where the $i$ th switch of load $j$, described by (4), (5), occurs.

Definition 1: The period of load $j$ is defined as $\pi_{j}=t_{j, i+2}-$ $t_{j, i}$, for any $i \geq 1$.

It should be clear that for any $j \in N$, it holds that $t_{j, i+2}-$ $t_{j, i}=t_{j, k+2}-t_{j, k}$, for all $i, k \in \mathbb{N}$. Note that, as follows from

${ }^{2}$ Note that the conditions $\hat{T}_{j}-\lambda_{j} \bar{d}_{j}<\underline{T}_{j}$ and $\hat{T}_{j}>\bar{T}_{j}$ correspond to cooling devices, such as air-conditioning units and refrigerators. These inequalities should be appropriately adapted for heating units, such as space heaters. This extension in the analysis is trivial and is hence omitted.

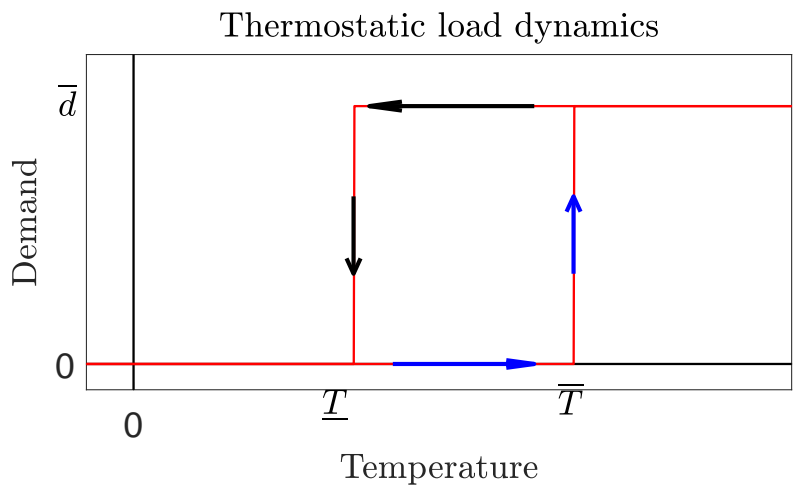

Fig. 1: TCL scheme described by (4).

(4), (5), the time lengths that load $j$ remains switched $\mathrm{ON}$ and OFF within each period are respectively given by

$$
\begin{gathered}
\pi_{j}^{O N}=\frac{1}{k_{j}} \ln \left(\frac{\bar{T}_{j}+\lambda_{j} \bar{d}_{j}-\hat{T}_{j}}{\underline{T}_{j}+\lambda_{j} \bar{d}_{j}-\hat{T}_{j}}\right), j \in N, \\
\pi_{j}^{O F F}=\frac{1}{k_{j}} \ln \left(\frac{\hat{T}_{j}-\underline{T}_{j}}{\hat{T}_{j}-\bar{T}_{j}}\right), j \in N,
\end{gathered}
$$

and that it trivially follows that $\pi_{j}=\pi_{j}^{O N}+\pi_{j}^{O F F}$. Furthermore, the duty cycle of each load is given by $\alpha_{j}=\frac{\pi_{j}^{O N}}{\pi_{j}}$, i.e. the ratio of time the load is $\mathrm{ON}$ within each period. Moreover, we define the period ratio between loads $i$ and $j$ as $\rho_{i j}=\frac{\pi_{i}}{\pi_{j}}$. We shall use $d_{j}^{c, *}=\alpha_{j} \bar{d}_{j}$ to denote the average value of $d_{j}^{c}$ when its dynamics are described by (4), (5). In addition, we let

$$
d^{s}=\sum_{j \in N} d_{j}^{c}, \quad \Gamma=\sum_{j \in N} \bar{d}_{j},
$$

be the aggregate sum and aggregate magnitude of TCLs, where $\Gamma \in \mathbb{R}_{+}$. Finally, we define $E=\{(i, j): i, j \in N, i \neq j\}$ as the set of all load pairs.

\section{B. Variance analysis}

In this section we consider the aggregation of TCLs and analyze its mean and variance. In particular, we study how the latter is influenced when the number of loads tends to infinity, assuming a constant aggregate sum.

An important assumption in the following analysis is that period ratios lie in the set $\mathbb{R}_{+} / \mathbb{Q}_{+}$. This is stated below.

Assumption 2: All loads $(i, j) \in E$ described by (4), (5), satisfy $\rho_{i j} \in \mathbb{R}_{+} / \mathbb{Q}_{+}$.

Assumption 2 is a technical condition that enables to deduce Theorem 1 below which shows that when the number of TCLs tends to infinity, then the variance of their aggregation is zero for any initial condition. In particular, when Assumption 2 holds, then $d^{s}$ is an aperiodic signal that exhibits variability in the time instances the individual loads switch on and off, thus leading to Theorem 1. Assumption2 excludes cases where two loads have identical periods, which makes the aggregation of any two loads periodic. The latter is true for all cases where $\rho_{i j} \in \mathbb{Q}_{+}$, which are hence excluded. Note that $\mathbb{Q}_{+}$is a set 
of measure zero and hence the condition $\rho_{i j} \in \mathbb{R}_{+} / \mathbb{Q}_{+}$is unlikely to be violated in practice.

The following theorem states that the variance of the aggregation of TCLs tends to zero as their number tends to infinity. Its proof can be found in the appendix.

Theorem 1: Consider thermostatic loads described by (4), (5), with $\bar{d}_{j}=\frac{\Gamma}{|N|}$ and let Assumption 2 hold. Then, $\mathbb{V}\left(d^{s}\right)<\frac{\Gamma^{2}}{|N|}$ and hence $\lim _{|N| \rightarrow \infty} \mathbb{V}\left(d^{s}\right)=0$.

Theorem 1 demonstrates that as the number of loads described by (4), (5), becomes large, then an almost flat aggregate demand should be expected, a desired feature to avoid large oscillations in the frequency response. Note that Theorem 1 as well as many of the results that follow, are stated for the case where $\bar{d}_{j}=\frac{\Gamma}{\mid N}, j \in N$, which suggests a constant aggregate sum $\Gamma$ and loads of identical magnitude. The assumption that all load magnitudes are identical is made for simplicity and could potentially be relaxed, as part of future work.

Remark 1: A result analogous to Theorem 1 could be obtained by adopting a stochastic description for TCLs, where these are modeled as independent random processes. Theorem 1 is stated based on the presented deterministic setting, described by (4)-(5), since it is used to prove the main results of the paper, which also consider deterministic dynamics.

\section{FREQUENCY CONTROL OF THERMOSTATIC LOADS}

In this section we present a frequency control scheme for TCLs and propose appropriate conditions for its design. For the proposed scheme, we show that, as the number of loads tends to infinity, then no synchronization phenomena occur and that there exist arbitrarily long time intervals where frequency deviations are arbitrarily small.

\section{A. Frequency control scheme for thermostatic loads}

We introduce in this subsection the frequency control policy for the TCLs, which is a scheme that provides an ancillary service at urgencies, i.e. when frequency deviations exceed particular thresholds. The scheme, depicted in Figure 2 is described below

$$
\begin{aligned}
& d_{j}^{c}=\bar{d}_{j} \sigma_{j}, \\
& \sigma_{j}\left(t^{+}\right)= \begin{cases}1, & \left\{\begin{array}{l}
T_{j} \geq \bar{T}_{j}, \\
\omega \geq \omega_{j}^{1} \text { and } T_{j} \geq \underline{T}_{j}+\epsilon_{j},
\end{array}\right. \\
0, \quad\left\{\begin{array}{l}
T_{j} \leq \underline{T}_{j}, \\
\omega \leq-\omega_{j}^{1} \text { and } T_{j} \leq \bar{T}_{j}-\epsilon_{j},
\end{array}\right. \\
\sigma_{j}(t),\left\{\begin{array}{l}
|\omega| \leq \omega_{j}^{1} \text { and } \underline{T}_{j} \leq T_{j} \leq \bar{T}_{j}, \\
\omega \leq-\omega_{j}^{1} \text { and } T_{j} \in\left[\bar{T}_{j}-\epsilon_{j}, \bar{T}_{j}\right], \\
\omega \geq \omega_{j}^{1} \text { and } T_{j} \in\left[\underline{T}_{j}, \underline{T}_{j}+\epsilon_{j}\right],
\end{array}\right.\end{cases}
\end{aligned}
$$

where $\omega_{j}^{1}>0$ are frequency thresholds and $0<\epsilon_{j}<\left(\bar{T}_{j}-\right.$ $\left.\underline{T}_{j}\right) / 2, j \in N$. Note that, $\epsilon_{j}$ in (8) serves to ensure than no Zeno behavior occurs as a result of the coupling between the frequency and TCL dynamics. The latter is analytically shown in Lemma 1 below.

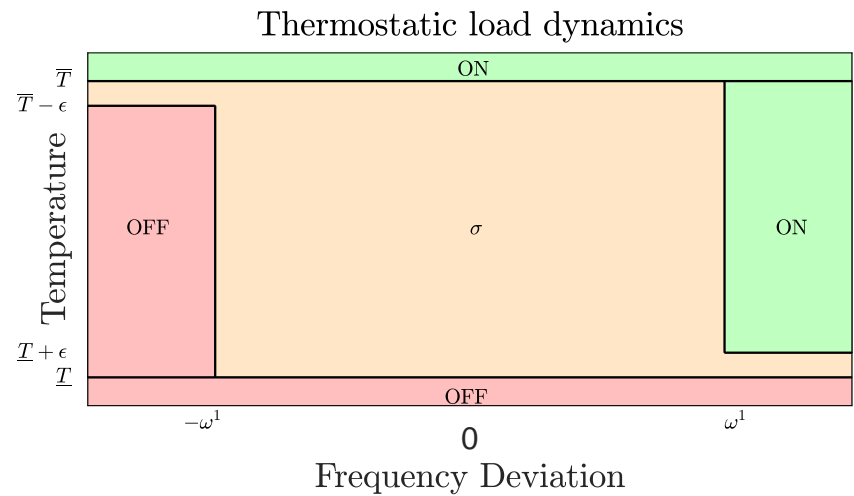

Fig. 2: TCL scheme described by (8). In the green and red areas the switching state is ON and OFF respectively. In the orange area the switching state can be either ON or OFF.

The scheme in (8) responds to frequency deviations by switching when prescribed frequency thresholds are exceeded thus providing ancillary services to the power network. Furthermore, when the frequency deviation does not reach the corresponding frequency thresholds, then the scheme in (8) reduces to (4). Note that, according to (8), the temperature will always be within its respective thresholds and hence users comfort levels will not be affected.

For the rest of the manuscript, we let $S(\bar{\omega})=\{j \in N$ : $\left.\omega_{j}^{1} \leq \bar{\omega}\right\}$ be the set of loads with respective frequency thresholds below $\bar{\omega}$. Moreover, for any set $S \subseteq N$, we let $\omega_{m}(S)=\min _{j \in S} \omega_{j}^{1}, d_{S}^{s}(t)=\sum_{j \in S} d_{j}^{c}(t)$ and $d_{S}^{s, *}=$ $\sum_{j \in S} \alpha_{j} \bar{d}_{j}$. Furthermore, we let $\hat{L}$ be the 1 -norm of the system with input $d^{s}$ and output $\omega$, described by (3), which is given by

$$
\hat{L}=\int_{0}^{\infty}\left|C \mathrm{e}^{A t} B\right| d t
$$

where $C=\left[\begin{array}{ll}1 & \mathbf{0}_{n}^{T}\end{array}\right]$, noting that its boundedness follows from Assumption 11i).

The following condition is imposed for the design of frequency thresholds. Within it, we let $\zeta_{j}=\max \left(\alpha_{j}, 1-\alpha_{j}\right)$, noting that $\zeta_{j} \in[0.5,1)$ since $\alpha_{j} \in(0,1)$.

Design condition 1: The frequency thresholds $\omega_{j}^{1}$ are chosen such that for all $\bar{\omega} \in \mathbb{R}_{+}$and some $\delta>0, \sum_{j \in S(\bar{\omega})} \zeta_{j} \bar{d}_{j} \leq$ $\max ((\bar{\omega}-\delta) / \hat{L}, 0)$, where $\hat{L}$ is given by (9).

Design condition 1 restricts the coupling of frequency and TCL dynamics by bounding the aggregate demand that actively contributes to frequency regulation. The condition allows to deduce that no synchronization occurs between TCLs when the scheme (8) is implemented. Note also that $\delta$ in Design condition 1 satisfies $\delta \in\left(0, \omega_{m}(N)\right)$ by definition, since $\omega_{j}^{1}<\delta$ for some $j \in N$ would imply that Design condition 11 does not hold. To implement Design condition 11. the values of $\omega^{1}$ for the TCL population should be selected such that the presented bound is satisfied at all values of $\bar{\omega}$, i.e. given $\bar{\omega}$, the condition restricts the aggregate demand of loads that may switch due to that particular frequency deviation. 


\section{B. Hybrid system description}

The behavior of system (1), (2), (5), (8), can be described by the states $z=(\bar{x}, \sigma)$, where $\bar{x}=(\omega, \hat{x}, T) \in \mathbb{R}^{m}, m=$ $|N|+n+1$, is the continuous state, and $\sigma \in P^{|N|}$ the discrete state, where $P=\{0,1\}$. Moreover, we let $\Lambda=\mathbb{R}^{m} \times P^{|N|}$ be the space where the system states evolve. The continuous dynamics of the system (1), (2), (5), (8), are described by

$$
\begin{gathered}
M \dot{\omega}=-p^{L}+p^{M}-D \omega-\sum_{j \in N} \bar{d}_{j} \sigma_{j}, \\
p^{M}=\hat{C} \hat{x}+\hat{D} \omega, \quad \dot{\hat{x}}=\hat{A} \hat{x}+\hat{B} \omega, \\
\dot{T}_{j}=-k_{j}\left(T_{j}-\hat{T}_{j}+\lambda_{j} \bar{d}_{j} \sigma_{j}\right), j \in N, \\
\dot{\sigma}_{j}=0, j \in N,
\end{gathered}
$$

which is valid when $z$ belongs to the set $F$ given by

$$
F=\left\{z \in \Lambda: \sigma_{j} \in \mathcal{I}_{j}\left(T_{j}, \omega\right), \forall j \in N\right\},
$$

where

$$
\mathcal{I}_{j}\left(T_{j}, \omega\right)=\left\{\begin{array}{l}
\{1\},\left\{\begin{array}{l}
T_{j}>\bar{T}_{j}, \\
\omega>\omega_{j}^{1} \text { and } T_{j}>\underline{T}_{j}+\epsilon_{j},
\end{array}\right. \\
\{0\},\left\{\begin{array}{l}
T_{j}<\underline{T}_{j}, \\
\omega<-\omega_{j}^{1} \text { and } T_{j}<\bar{T}_{j}-\epsilon_{j},
\end{array}\right. \\
\{0,1\},\left\{\begin{array}{l}
|\omega| \leq \omega_{j}^{1} \text { and } \underline{T}_{j} \leq T_{j} \leq \bar{T}_{j}, \\
\omega \leq-\omega_{j}^{1} \text { and } T_{j} \in\left[\bar{T}_{j}-\epsilon_{j}, \bar{T}_{j}\right], \\
\omega \geq \omega_{j}^{1} \text { and } T_{j} \in\left[\underline{T}_{j}, \underline{T}_{j}+\epsilon_{j}\right] .
\end{array}\right.
\end{array}\right.
$$

Alternatively, when $z$ belongs to the set $G=(\Lambda \backslash F) \cup \underline{G}$ where $\underline{G}=\left\{z \in \Lambda: \sigma_{j} \in \mathcal{I}_{j}^{D}\left(T_{j}, \omega\right), \forall j \in N\right\}$, and

$$
\mathcal{I}_{j}^{D}\left(T_{j}, \omega\right)=\left\{\begin{aligned}
&\{1\},\left\{\begin{array}{l}
\omega \geq-\omega_{j}^{1} \text { and } T_{j}=\underline{T}_{j}, \\
\omega=-\omega_{j}^{1} \text { and } T_{j} \in\left[\underline{T}_{j}, \bar{T}_{j}-\epsilon_{j}\right], \\
\omega \leq-\omega_{j}^{1} \text { and } T_{j}=\bar{T}_{j}-\epsilon_{j},
\end{array}\right. \\
&\{0\},\left\{\begin{array}{l}
\omega \leq \omega_{j}^{1} \text { and } T_{j}=\bar{T}_{j}, \\
\omega=\omega_{j}^{1} \text { and } T_{j} \in\left[\underline{T}_{j}+\epsilon_{j}, \bar{T}_{j}\right], \\
\omega \geq \omega_{j}^{1} \text { and } T_{j}=\underline{T}_{j}+\epsilon_{j},
\end{array}\right.
\end{aligned}\right.
$$

then its components follow the discrete update described below

$$
\bar{x}^{+}=\bar{x}(t), \sigma_{j}\left(t^{+}\right)=\left\{\begin{array}{l}
1,\left\{\begin{array}{l}
T_{j} \geq \bar{T}_{j}, \\
\omega \geq \omega_{j}^{1} \text { and } T_{j} \in\left[\underline{T}_{j}+\epsilon_{j}, \bar{T}_{j}\right],
\end{array}\right. \\
0,\left\{\begin{array}{l}
T_{j} \leq \underline{T}_{j}, \\
\omega \leq-\omega_{j}^{1} \text { and } T_{j} \in\left[\underline{T}_{j}, \bar{T}_{j}-\epsilon_{j}\right],
\end{array}\right.
\end{array}\right.
$$

where $\bar{x}^{+}=\lim _{\epsilon \rightarrow 0} \bar{x}(t+\epsilon)$.

We can now provide the following compact representation for the hybrid system (1), (2), (5), (8),

$$
\dot{z}=f(z), z \in F, \quad z^{+}=g(z), z \in G,
$$

where $f(z): F \rightarrow \Lambda$ and $g(z): G \rightarrow F$ are described by (10) and (12) respectively. Note that $z^{+}=g(z)$ represents a discrete dynamical system where $z^{+}$indicates that the next value of the state $z$ is given as a function of its current value through $g(z)$. Moreover, notice that $F \cup G=\Lambda$.

\section{Analysis of solutions}

In this section we consider the solutions of (13) and show their existence and that no Zeno behavior occurs. Below we provide a definition of a hybrid time domain, hybrid solution and complete and maximal solutions for systems described by (13) from [25, Ch. 2]. Note that the definition of a hybrid system is provided in [25, Dfn. 2.2].

Definition 2: ( [25]) A subset of $\mathbb{R}_{\geq 0} \times \mathbb{N}_{0}$ is a hybrid time domain if it is a union of a finite or infinite sequence of intervals $\left[t_{\ell}, t_{\ell+1}\right] \times\{\ell\}$, with the last interval (if existent) possibly of the form $\left[t_{\ell}, t_{\ell+1}\right] \times\{\ell\},\left[t_{\ell}, t_{\ell+1}\right) \times\{\ell\}$, or $\left[t_{\ell}, \infty\right) \times\{\ell\}$. Consider a function $z(t, \ell): K \rightarrow \mathbb{R}^{m}$ defined on a hybrid time domain $K$ such that for every fixed $\ell \in \mathbb{N}$, $t \rightarrow z(t, \ell)$ is locally absolutely continuous on the interval $T_{\ell}=\{t:(t, \ell) \in K\}$. The function $z(t, \ell)$ is a solution to the hybrid system $\mathcal{H}=(F, f, G, g)$ if $z(0,0) \in F \cup G$, and for all $\ell \in \mathbb{N}$ such that $T_{\ell}$ has non-empty interior (denoted by $\operatorname{int} T_{l}$ )

$$
\begin{aligned}
& z(t, \ell) \in F, \text { for all } \mathrm{t} \in \operatorname{int} T_{l}, \\
& \dot{z}(t, \ell) \in f(z(t, \ell)), \text { for almost all } t \in T_{\ell},
\end{aligned}
$$

and for all $(t, \ell) \in K$ such that $(t, \ell+1) \in K$,

$$
z(t, \ell) \in G, z(t, \ell+1) \in g(z(t, \ell)) .
$$

A solution $z(t, \ell)$ is complete if $K$ is unbounded. A solution $z$ is maximal if there does not exist another solution $\tilde{z}$ with time domain $\tilde{K}$ such that $K$ is a proper subset of $\tilde{K}$ and $z(t, j)=\tilde{z}(t, j)$ for all $(t, j) \in K$.

The following lemma, proven in the appendix, shows the existence of complete solutions to (13). Furthermore, it demonstrates the boundedness of solutions to (13) and provides a lower bound on the time between consecutive switches, which suffices to show that no Zeno behavior occurs. Finally, it states that all maximal solutions to (13) are complete. We remind that $t_{j, i}$ is the time of the $i$ th switch of load $j$.

Lemma 1: For any initial condition $z(0,0) \in \Lambda$ there exists a complete solution to (13). Furthermore, all maximal solutions to (13) are complete. Moreover, if Assumption 1 holds then the following hold:

(i) For each initial condition $z(0,0) \in \Lambda$, solutions to (13) are bounded.

(ii) For any solution to (13), there exists $\tau_{d}>0$ such that $\min _{i \geq 1}\left(t_{j, i+1}-t_{j, i}\right) \geq \tau_{d}$ for any $j \in N$.

The boundedness of solutions to (13), demonstrated in the above lemma, follows also intuitively by noting that (13) consists of the asymptotically stable linear system (1), (2), with input $d^{s}$ and output $\omega$ in feedback with the hybrid system (5), (8) and that the magnitude of $d^{s}$, which can be regarded as the output of (5), (8), is bounded. Furthermore, the boundedness of $T_{j}, j \in N$ follows directly from the structure of (5), (8).

\section{Performance analysis}

In this section we state one of the main results of this paper, associated with the performance of solutions to (13). The following theorem, proven in the appendix, demonstrates that as the number of loads tends to infinity, then for all initial 
conditions there exist arbitrarily long time intervals where frequency deviations are arbitrarily small.

Theorem 2: Consider the system described by (13) and let Assumptions 1 12 and Design condition 11 hold. Furthermore, assume that the thermostatic loads described by (5), (8) satisfy $\bar{d}_{j}=\frac{\Gamma}{|N|}$. Then, as $|N| \rightarrow \infty$, for any $z(0,0) \in \mathbb{R}^{m} \times P^{|N|}$, any maximal solution of (13) and any $\epsilon>0, \hat{\tau} \in \mathbb{R}_{+}$, there exists $\tau \in \mathbb{R}_{+}$such that $|\omega(t, j)| \leq \epsilon$ for $t \in[\tau, \tau+\hat{\tau}]$.

The importance of Theorem 2 is that it shows, for all initial conditions, that frequency trajectories become arbitrarily small for an arbitrarily long amount of time. Also, as shown in Lemma 1 the scheme in (8) avoids Zeno behavior. Furthermore, being deterministic, it allows the instant response to frequency deviations, thus providing improved ancillary services to the power system. The latter, is also numerically demonstrated in the following section.

Remark 2: Theorem 2] does not provide an analytical expression for $\tau$. However, it is intuitive to note that its value in a real setting depends on: (i) the values of $\epsilon$ and $\hat{\tau}$, which are associated with its definition, (ii) the initial conditions and the speed of generation dynamics, which determine how long it takes for generation to match a potential disturbance, and (iii) the distribution of load periods.

\section{Simulation ON THE NPCC 140-BUS SYSTEM}

In this section we verify our analytic results with a numerical simulation on the Northeast Power Coordinating Council (NPCC) 140-bus interconnection system, using the Power System Toolbox [26]. This model is more detailed and realistic than our analytical one, including line resistances, a DC12 exciter model, a transient reactance generator model, and turbine governor dynamics.

The test system consists of 93 load buses serving different types of loads including constant active and reactive loads and 47 generation buses. The overall system has a total real power of $28.55 \mathrm{GW}$. For our simulation, we added five loads on buses $2,8,9,16$ and 17 , each having a step increase of magnitude 2 p.u. (base 100MVA) at $t=1$ second.

Controllable loads were considered within the simulations at load buses $1-20$, with loads controlled every $10 \mathrm{~ms}$. In particular, we considered 500 refrigerators of equal magnitude at each of the 20 selected load buses with aggregate power of $2.5 \mathrm{GW}$. For comparison, we considered the system response when the following four schemes for TCLs were implemented.

(i) Conventional TCLs that do not contribute to frequency control, i.e. loads with dynamics as in (4), (5).

(ii) Frequency dependent TCLs with a deterministic control policy, i.e. loads with dynamics described by (5), (8).

(iii) Frequency dependent TCLs with a randomized control policy, as in [16], [17].

(iv) The scheme (iii) with larger feedback gains, aiming for a faster response.

The above cases will be referred to as case (i), (ii), (iii) and (iv) respectively. The values of the control parameters were

\footnotetext{
${ }^{3} \mathrm{~A}$ more realistic simulation would involve $10^{6}$ refrigerators for the same aggregate demand but would be computationally expensive. The simulated number suffices to demonstrate the analysis in the paper noting that a larger number of TCLs would result in an even smoother response.
}

\begin{tabular}{|l|l|l|}
\hline Variable & Lower Bound & Upper Bound \\
\hline \hline$\hat{T}$ & $15^{\circ} \mathrm{C}$ & $25^{\circ} \mathrm{C}$ \\
\hline $\bar{T}$ & $5^{\circ} \mathrm{C}$ & $7^{\circ} \mathrm{C}$ \\
\hline$\underline{T}$ & $2^{\circ} \mathrm{C}$ & $4{ }^{\circ} \mathrm{C}$ \\
\hline$k$ & $2 \times 10^{-4}$ & $10^{-3}$ \\
\hline$\lambda_{j}$ & $25\left(\bar{d}_{j}\right)^{-1}$ & $35\left(\bar{d}_{j}\right)^{-1}$ \\
\hline$\omega^{1}$ & $0.01 \mathrm{~Hz}$ & $0.26 \mathrm{~Hz}$ \\
\hline$\epsilon_{j}$ & $0.001{ }^{\circ} \mathrm{C}$ & $0.01{ }^{\circ} \mathrm{C}$ \\
\hline
\end{tabular}

TABLE I: Ranges of coefficients describing TCL dynamics.

randomly selected from uniform distributions with bounds provided in Table [. Furthermore, initial conditions were randomly selected in a similar manner. To ensure that incorporating the loads would not disturb the balance of the network, for each thermostatic load incorporated at a bus some constant demand equal to its average value was removed from the same bus. Moreover, frequency thresholds in case (ii) were selected in accordance with Design condition 1 In particular, following the approach described in [22], an equivalent single bus model of the power network, where generation was described with high order dynamics, was derived. The latter enabled to obtain $\hat{L}$ (i.e. the 1-norm of the system (3) with input the aggregate demand and output $\omega$ ) and implement Design condition 11 To ensure that Design condition 11 was satisfied, we verified that the selected values of $\omega^{1}$ satisfied $\sum_{j \in S(\bar{\omega})} \zeta_{j} \bar{d}_{j} \leq \max ((\bar{\omega}-\delta) / \hat{L}, 0)$, letting $\delta=0.001 H z$, for all $\bar{\omega} \in \mathbb{R}_{+}$. For additional safety, frequency thresholds were designed with a $20 \%$ margin from the obtained upper bound. For case (iii), the implemented algorithm involved randomized transitions between the on/off states with controlled rates 4 as in [16]. The algorithm was implemented with $K_{\pi}=5$ and $v_{\text {des }}=1$ for each TCL (in analogy to [16]), where $K_{\pi}$ and $v_{\text {des }}$ are parameters associated with the feeback gain and the desired temperature variability respectively. For case (iv), we implemented case (iii) with $K_{\pi}=50$.

The frequency at bus 27 for the four tested cases is shown in Figure 3 . We observe that the frequency converges to a very small set containing its nominal value. Furthermore, Figure 4 suggests that the scheme in (8) results in a reduced frequency overshoot relative to the other cases considered, by illustrating the largest deviation in frequency at buses $1-40$, which are the buses where the frequency overshoot was seen to be the largest. In addition, it demonstrates that increasing the feedback gains in the schemes with randomization in cases (iii), (iv) results in a reduced frequency overshoot. However, larger transition rates can lead to more frequent switching of the TCLs, which is generally undesirable. Furthermore, increasing only one of the transition rates (as is the effect of increasing $K_{\pi}$ ) will maintain a slower recovery of the TCLs. The speed of response of schemes with randomization can potentially be improved by combining them with deterministic schemes, which is an interesting direction for further theoretical analysis. Figures 3, 4 also demonstrate that no Zeno behavior or load synchronization are experienced with the proposed deterministic scheme.

\footnotetext{
${ }^{4}$ Additional temperature constraints were not considered for simplicity, as simulations indicate that these restrict the frequency control performance when the temperature thresholds are fixed.
} 


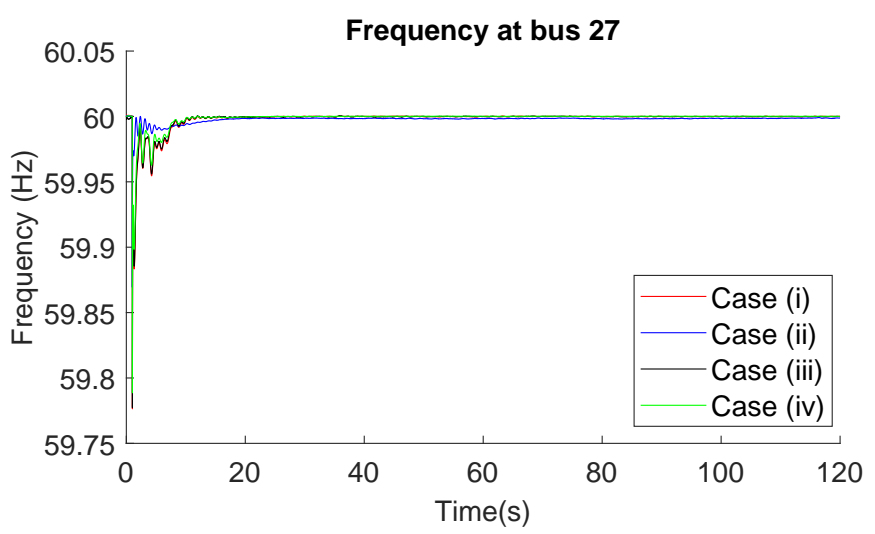

Fig. 3: Frequency at bus 27 with TCL dynamics in the following cases: i) Conventional TCLs, ii) Deterministic frequency dependent TCLs, iii) Frequency dependent TCLs with randomized control policy, iv) As in (iii) but feedback gains are ten times larger.

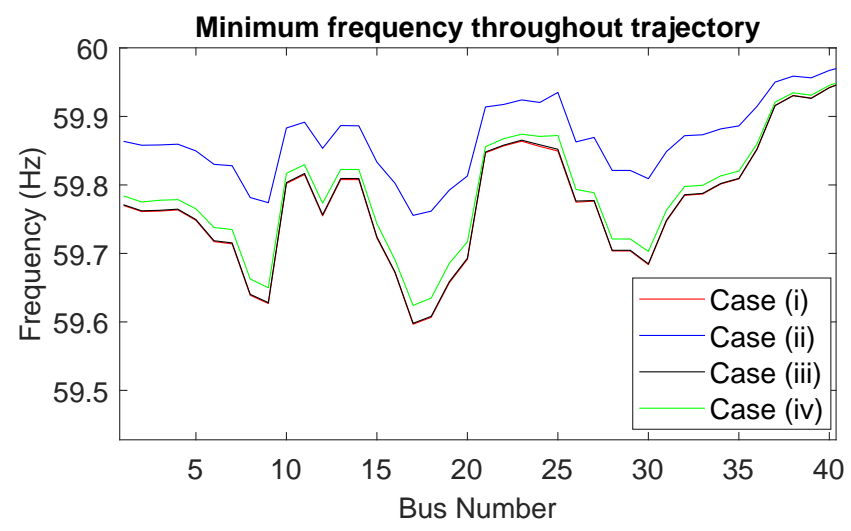

Fig. 4: Largest frequency overshoot for buses $1-40$ for the four cases described in the caption of Figure 3

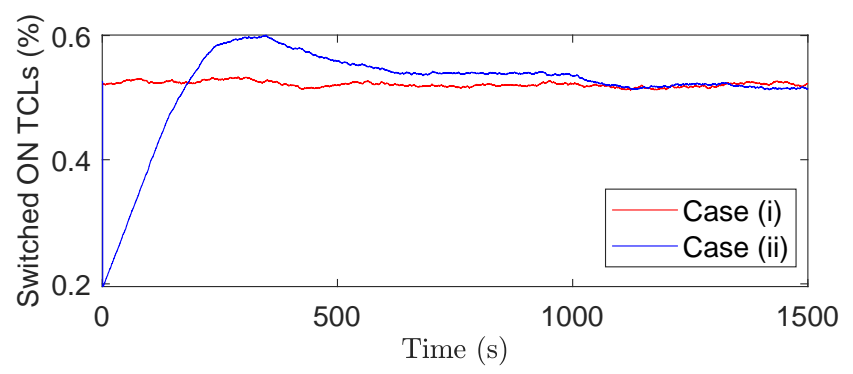

Fig. 5: Percentage of TCLs switched ON for the following cases: i) Conventional TCLs, ii) Deterministic frequency dependent TCLs.

The percentage of TCLs that are ON for the TCL schemes described in cases (i) and (ii) is depicted in Figure 5. It should be noted that the almost flat response in case (i) validates Theorem 1 .

\section{CONCLUSION}

We have studied the problem of controlling thermostatic loads to provide ancillary services to the power network at urgencies. We first considered conventional TCLs and showed that their aggregation has zero variance when their number tends to infinity and a mild condition on their period ratios holds. Then, we proposed a deterministic control scheme for TCLs which induces switching when frequency deviations exceed particular frequency thresholds. For the considered scheme, we explain how frequency thresholds could be designed such that the coupling between load and frequency dynamics does not cause load synchronization. In particular, when the number of loads tends to infinity, we showed that frequency deviations are arbitrarily small for arbitrarily large periods of time. Our analytic results have been numerically verified with simulations on the NPCC 140-bus system, which demonstrate improved frequency response when frequency dependent TCLs are incorporated compared to when conventional implementations are considered.

Future extensions of this work could consider more involved dynamics, including a network model of the power grid. In addition, future studies could consider more advanced control designs for TCLs, taking into account elements such as their economic performance and the rate at which they desynchronize, which may yield improved response.

\section{APPENDIX}

Proof of Theorem 1 . By definition, the variance is given by

$$
\mathbb{V}\left(d^{s}\right)=\mathbb{E}\left(\left(d^{s}\right)^{2}\right)-\left[\mathbb{E}\left(d^{s}\right)\right]^{2} .
$$

Since $d^{s}=\sum_{j \in N} d_{j}^{c}$, it then holds that

$$
\begin{aligned}
\mathbb{E}\left(\left(d^{s}\right)^{2}\right) & =\sum_{j \in N} \mathbb{E}\left(\left(d_{j}^{c}\right)^{2}\right)+2 \sum_{(i, j) \in E} \mathbb{E}\left(d_{i}^{c} d_{j}^{c}\right) \\
& =\sum_{j \in N} \alpha_{j}\left(\frac{\Gamma}{|N|}\right)^{2}+2 \sum_{(i, j) \in E} \alpha_{i} \alpha_{j}\left(\frac{\Gamma}{|N|}\right)^{2},
\end{aligned}
$$

where the first argument follows trivially and the second from Proposition 1 below. Furthermore, the second term in (14) satisfies

$$
\begin{aligned}
& {\left[\mathbb{E}\left(d^{s}\right)\right]^{2}=\left[\mathbb{E}\left(\sum_{j \in N} d_{j}^{c}\right)\right]^{2}=\sum_{j \in N} \mathbb{E}\left(d_{j}^{c}\right)^{2}+2 \sum_{(i, j) \in E} \mathbb{E}\left(d_{i}^{c}\right) \mathbb{E}\left(d_{j}^{c}\right)} \\
& =\sum_{j \in N} \alpha_{j}^{2}\left(\frac{\Gamma}{|N|}\right)^{2}+2 \sum_{(i, j) \in E} \alpha_{i} \alpha_{j}\left(\frac{\Gamma}{|N|}\right)^{2} . \\
& \text { Combining (14), 15 and (16) results to } \mathbb{V}\left(d^{s}\right)=
\end{aligned}
$$
$\sum_{j \in N} \alpha_{j}\left(1-\alpha_{j}\right)\left(\frac{\Gamma}{|N|}\right)^{2}<\frac{\Gamma^{2}}{|N|}$ noting for the last argument that $0<\alpha_{j}<1, j \in N$. Hence, it holds that $\lim _{|N| \rightarrow \infty} \mathbb{V}\left(d^{s}\right)=0$.

Proposition 1: Consider TCLs described by (4), (5) and let Assumption 2 hold. Then, $\mathbb{E}\left(d_{i}^{c} d_{j}^{c}\right)=\alpha_{i} \alpha_{j} \Gamma_{i} \Gamma_{j}$ for all $(i, j) \in E$.

Proof of Proposition 17. From Assumption 2] it follows that $\rho_{i j} \in \mathbb{R}_{+} / \mathbb{Q}_{+}$for all $(i, j) \in E$ and hence the signal $d_{i}^{c}(t) d_{j}^{c}(t)$ is aperiodic. Its average is defined as

$$
\mathbb{E}\left(d_{i}^{c} d_{j}^{c}\right)=\lim _{\tau \rightarrow \infty} \frac{1}{\tau} \int_{0}^{\tau} d_{i}^{c}(t) d_{j}^{c}(t) d t .
$$


Without loss of generality let $\pi_{i}>\pi_{j}$ and $t_{i, 1}, t_{j, 1}$ be the first time when loads $i$ and $j$ switch ON respectively. Then, let $\hat{t}_{k}=\left[t_{j, 1}-t_{i, 1}-(k-1) \pi_{i}\right]_{\pi_{j}}^{+}$noting that it represents the time difference between the $k$-th time load $i$ switches $\mathrm{ON}$ and the first time load $j$ switches ON afterwards. Then, letting 5 $c_{k}=\left[\hat{t}_{k+1}-\hat{t}_{k}\right]_{\pi_{j}}^{+}=\left[-\pi_{i}\right]_{\pi_{j}}^{+}$, it follows that $c_{k}=c, \forall k \geq 1$. Furthermore, since $c=\mu \pi_{j}-\pi_{i}$, for some $\mu \in \mathbb{N}$ and $\rho_{i j} \in$ $\mathbb{R}_{+} / \mathbb{Q}_{+}$, it follows that $\frac{c}{\pi_{j}} \in \mathbb{R}_{+} / \mathbb{Q}_{+}$. Hence, (17) satisfies

$$
\mathbb{E}\left(d_{i}^{c} d_{j}^{c}\right)=\frac{1}{\pi_{i}} \lim _{\hat{N} \rightarrow \infty} \frac{1}{\hat{N}} \sum_{k=1}^{\hat{N}} \int_{0}^{\pi_{i}} d_{i}^{c}(t) d_{j}^{c}\left(t-\hat{t}_{k}\right) d t .
$$

From the definition of $\hat{t}_{k}$ it follows that its values lie within $\left[0, \pi_{j}\right]$. Furthermore, from $c_{k}=c, k \geq 1$ and $\frac{c}{\pi_{j}} \in \mathbb{R}_{+} / \mathbb{Q}_{+}$, it follows that the sequence of $\left[\hat{t}_{k}\right]_{\pi_{j}}^{+}$ becomes uniformly distributed as $k \rightarrow \infty$. The latter follows by noting that the sequence $\left\{\left[\hat{t}_{2}-\hat{t}_{1}\right]_{\pi_{j}}^{+},\left[\hat{t}_{3}-\right.\right.$ $\left.\left.\hat{t}_{1}\right]_{\pi_{j}}^{+},\left[\hat{t}_{4}-\hat{t}_{1}\right]_{\pi_{j}}^{+}, \ldots,\left[\hat{t}_{N+1}-\hat{t}_{1}\right]_{\pi_{j}}^{+}, \ldots\right\}$, which is equal to $\left\{[c]_{\pi_{j}}^{+},[2 c]_{\pi_{j}}^{+},[3 c]_{\pi_{j}}^{+}, \ldots,[N c]_{\pi_{j}}^{+}, \ldots\right\}$, is equivalent to the sequence $\pi_{j} \times\left\{\left[\frac{c}{\pi_{j}}\right]_{1}^{+},\left[\frac{2 c}{\pi_{j}}\right]_{1}^{+},\left[\frac{3 c}{\pi_{j}}\right]_{1}^{+}, \ldots,\left[\frac{N c}{\pi_{j}}\right]_{1}^{+}, \ldots\right\}$ and the uniformity of this sequence is a special case of the Weyl Criterion (e.g. [28, Theorem 2.1]) since $\frac{c}{\pi_{j}}$ is irrational. From the last argument it follows that (18) can be equivalently written as

$$
\mathbb{E}\left(d_{i}^{c} d_{j}^{c}\right)=\frac{1}{\pi_{i} \pi_{j}} \int_{0}^{\pi_{j}} \int_{0}^{\pi_{i}} d_{i}^{c}(t) d_{j}^{c}\left(t-\bar{t}_{1}\right) d t d \bar{t}_{1} .
$$

Then, considering that for $t \in\left[0, \pi_{i}\right]$ it holds that

$$
d_{i}^{c}(t)=\left\{\begin{array}{l}
\Gamma_{i}, t \in\left[t_{i, 1}, t_{i, 1}+\alpha_{i} \pi_{i}\right] \\
0, \text { otherwise }
\end{array}\right.
$$

and defining $s=\max _{\gamma \in \mathbb{N}_{0}}\left\{\gamma: \alpha_{i} \pi_{i}>\gamma \pi_{j}\right\}$, it follows that

$$
\mathbb{E}\left(d_{i}^{c} d_{j}^{c}\right)=\frac{\Gamma_{i}}{\pi_{i} \pi_{j}} \int_{0}^{\pi_{j}}\left[s \pi_{j} \alpha_{j} \Gamma_{j}+\int_{t_{i, 1}+s \pi_{j}}^{t_{i, 1}+\alpha_{i} \pi_{i}} d_{j}^{c}\left(t-\bar{t}_{1}\right) d t\right] d \bar{t}_{1} .
$$

The second integral in (19) can be evaluated as

$$
\int_{0}^{\pi_{j}} \int_{t_{i, 1}+s \pi_{j}}^{t_{i, 1}+\alpha_{i} \pi_{i}} d_{j}^{c}\left(t-\bar{t}_{1}\right) d t d \bar{t}_{1}=\pi_{j}\left(\alpha_{i} \pi_{i}-s \pi_{j}\right) \alpha_{j} \Gamma_{j},
$$

which from (19) results to $\mathbb{E}\left(d_{i}^{c} d_{j}^{c}\right)=\alpha_{i} \alpha_{j} \Gamma_{i} \Gamma_{j}$.

Proof of Lemma 1 . The existence of a complete solution to (13) follows trivially from the fact that the dynamics in (10) are globally Lipschitz and that $f$ and $g$ map into $\Lambda$ which is the domain of (13). Furthermore, the fact that all maximal solutions to (13) are complete follows from the global Lipschitz property of $f$ and the fact that $f$ and $g$ map into $\Lambda$ which is the domain of (13) [25, Proposition 6.10]. The rest two parts of the Lemma are proved below:

(i) The boundedness of $\left(\omega, p^{M}\right)$ and $T_{j}, j \in N$ follows since 10a-10b and 10c can be seen as asymptotically stable linear systems (Assumption 11(i)) with bounded inputs $\Sigma_{j \in N} d_{j}^{c} \sigma_{j}$ and $d_{j}^{c} \sigma_{j}$ respectively.

\footnotetext{
${ }^{5}$ The fact that $\left[\hat{t}_{k+1}-\hat{t}_{k}\right]_{\pi_{j}}^{+}=\left[-\pi_{i}\right]_{\pi_{j}}^{+}$follows from the modular addition property, which is a standard property in modular arithmetics, see e.g. [27. Ch. 4.1].
}

(ii) From (i) note that for each $z(0,0) \in \Lambda$, the solution to system (13), with states $z=\left(\omega, p^{M}, T, \sigma\right)$, is bounded. Then, note that the values of $\dot{\omega}$ and $\dot{T}_{j}$ are bounded from above by constants, $d \omega^{\max }$ and $d T_{j}^{\max }$, as a result of the boundedness of solutions and the fact that the vector field in (10) is globally Lipschitz. Hence, it follows that $t_{j, \ell+1}-t_{j, \ell} \geq \min \left(2 \omega_{j}^{1} / d \omega_{j}^{\max }, \epsilon_{j} / d T_{j}^{\max }\right)=\tau_{j}$. Finally, let $\tau_{d}=\min _{j \in N} \tau_{j}$ to conclude the proof.

The following results will be used within the proof of Theorem 2

Corollary 1: Let Assumption 2 hold and consider TCLs described by (5), (8) with $\bar{d}_{j}=\frac{\Gamma}{|N|}$ and any set $S \subseteq N$. Then, if there exists $\tau \geq 0$ such that $|\omega(t)|<\omega_{m}(S)$ for all $t \geq \tau$ then $\mathbb{V}\left(d_{S}^{s}\right) \rightarrow 0$ as $|N| \rightarrow \infty$.

Proof of Corollary 7 . When for some finite $\tau$ it holds that $|\omega(t)|<\omega_{j}^{1}, t \geq \tau, j \in S$, the scheme in (8) reduces to (4) for $j \in S$. If $|S| \rightarrow \infty$ as $|N| \rightarrow \infty$, the proof follows directly from Theorem 1 and the boundedness of $d_{S}^{s}$. Alternatively, if $|S|<\infty$ as $|N| \rightarrow \infty$, then the proof follows trivially by noting that $\lim _{|N| \rightarrow \infty} \sum_{j \in S} d_{j}^{c} \leq \lim _{|N| \rightarrow \infty}|S| \frac{\Gamma}{|N|}=0$.

Lemma 2: Consider TCLs described by (5), (8), with $\bar{d}_{j}=$ $\frac{\Gamma}{|N|}$, any set $S \subseteq N$ and let Assumption 2 hold. Then, when $|N| \rightarrow \infty$, for any initial condition $(T(0), \sigma(0)) \in \mathbb{R}^{|N|} \times$ $P^{|N|}$ and any $\epsilon>0, \hat{\tau}_{1} \in \mathbb{R}_{+}$, there exist $\tau, \tau_{1}, \hat{\tau}_{0} \in \mathbb{R}_{+}, \tau \leq$ $\tau_{1}, \tau_{1}+\hat{\tau}_{1} \leq \hat{\tau}_{0}$ such that if $|\omega(t)|<\omega_{m}(S)$ for $t \in\left[\tau, \hat{\tau}_{0}\right]$, then $\int_{\tau_{1}}^{\tau_{1}+\hat{\tau}_{1}}\left(d_{S}^{s}(t)-d_{S}^{s, *}\right)^{2} d t \leq \epsilon$.

Proof of Lemma 2. From Theorem 1 it follows that when Assumption 2 holds for TCLs described by (4), (5), with $\bar{d}_{j}^{c}=\frac{\Gamma}{|N|}$ then $\lim _{|N| \rightarrow \infty} \mathbb{V}\left(d^{s}\right)=0$. Corollary 1 extends this result to any set $S \subseteq N$, i.e. $\lim _{|N| \rightarrow \infty} \mathbb{V}\left(d_{S}^{s}\right)=0$. The latter suggests that $\lim _{|N| \rightarrow \infty} \lim _{\tau \rightarrow \infty} \frac{1}{\tau} \int_{0}^{\tau}\left(d_{S}^{s}(t)-d_{S}^{s, *}\right)^{2} d t=0$ which follows since

$$
\begin{aligned}
& \lim _{\tau \rightarrow \infty} \frac{1}{\tau} \int_{0}^{\tau}\left(d_{S}^{s}(t)-d_{S}^{s, *}\right)^{2} d t=\lim _{\tau \rightarrow \infty} \frac{1}{\tau} \int_{0}^{\tau}\left(d_{S}^{s}(t)\right)^{2}-\left(d_{S}^{s, *}\right)^{2} d t \\
& =\lim _{\tau \rightarrow \infty} \frac{1}{\tau} \int_{0}^{\tau}\left(d_{S}^{s}(t)\right)^{2} d t-\left(d_{S}^{s, *}\right)^{2} \equiv \mathbb{V}\left(d_{S}^{s}\right),
\end{aligned}
$$

where the first step follows by expanding the squared term and using the definition of $d_{S}^{S, *}$.

Now consider the condition on the lemma statement, and temporarily assume that $|\omega(t)|<\omega_{m}(S)$ for all $t \geq \tau$. Therefore, (5), (8) reduces to (4), (5) for $t \geq \tau$.

The next part of the proof follows by contradiction. In particular, assume there exist $\epsilon>0$ and $\tau_{1}, \hat{\tau}_{1} \in \mathbb{R}_{+}$such that $\int_{\bar{\tau}}^{\bar{\tau}+\hat{\tau}_{1}}\left(d_{S}^{s}(t)-d_{S}^{s, *}\right)^{2} d t \geq \epsilon$ for all $\bar{\tau} \in\left\{\tau_{1}+k \hat{\tau}_{1}\right.$ : $\left.k \in \mathbb{N}_{0}\right\}$. Then, $\mathbb{V}\left(d_{S}^{s}\right) \geq \frac{\epsilon}{\hat{\tau}_{1}}$, which contradicts the result of Theorem 1 Hence, if $|\omega(t)|<\omega_{m}(S)$ for all $t \geq \tau$, then for any $\epsilon>0, \hat{\tau}_{1} \in \mathbb{R}_{+}$, there exists finite $\tau_{1}$ such that $\int_{\tau_{1}}^{\tau_{1}+\hat{\tau}_{1}}\left(d_{S}^{s}(t)-d_{S}^{s, *}\right)^{2} d t \leq \epsilon$.

To conclude the proof, note that the trajectory of $d^{s}(t)$ depends only on the initial conditions and the trajectory of $\omega(t)$. Hence, the trajectory of $\omega(t)$ for $t \geq \tau_{1}+\hat{\tau}_{1}$ does not affect the fact the result that $\int_{\tau_{1}}^{\tau_{1}+\hat{\tau}_{1}}\left(d_{S}^{s}(t)-d_{S}^{s, *}\right)^{2} d t \leq \epsilon$. Therefore, the condition on $\omega(t)$ reduces to $|\omega(t)|<\omega_{m}(S)$ for $t \in\left[\tau, \hat{\tau}_{0}\right]$, for any $\hat{\tau}_{0} \geq \tau_{1}+\hat{\tau}_{1}$.

Before continuing with the rest of the results, it will be convenient to note that system (13) consists of the linear system 
(3) in feedback with the hybrid system (5), (8). Let $\hat{x}^{*}$ be the equilibrium value of $\hat{x}$ in (3) when $d^{s}=d^{s, *}=\sum_{j \in N} \alpha_{j} \bar{d}_{j}$. System (3), can be equivalently written in terms of deviations from these equilibrium values as follows

$$
y=C x, \quad \dot{x}=A x+B u,
$$

where $x=\left[\begin{array}{c}\omega \\ \hat{x}-\hat{x}^{*}\end{array}\right], u=\left[d^{s}-d^{s, *}\right], y=\omega, C=\left[\begin{array}{ll}1 & \mathbf{0}_{n}^{T}\end{array}\right]$ and $A$ and $B$ as given in the description immediately after (3). Furthermore, note that $A$ is Hurwitz from Assumption 11(i).

Lemma 3: Consider the system (21). Let $|u(t)|$ be uniformly bounded for $t \geq 0$ and satisfy the property that for any $\epsilon>$ $0, \hat{\tau}_{1} \in \mathbb{R}_{+}$, there exists $\tau_{1} \in \mathbb{R}_{+}$such that $\int_{\tau_{1}}^{\tau_{1}+\hat{\tau}_{1}} u^{2} d t \leq \epsilon$. Then, for any $x(0) \in \mathbb{R}^{n+1}$ and any $\hat{\epsilon}>0, \bar{\tau}_{1} \in \mathbb{R}_{+}$, there exists $\tilde{\tau}_{1} \in \mathbb{R}_{+}$such that $|y(t)| \leq \hat{\epsilon}$ for all $t \in\left[\tilde{\tau}_{1}, \tilde{\tau}_{1}+\bar{\tau}_{1}\right]$.

Proof of Lemma 3. By assumption, given $\bar{\tau}_{1}$, and any $\epsilon>$ 0 , there exists $\hat{\tau}_{1}>\bar{\tau}_{1}$ for which there exists $\tau_{1}$ such that $\int_{\tau_{1}}^{\tau_{1}+\hat{\tau}_{1}} u^{2} d t \leq \epsilon$. Furthermore, $x\left(\tau_{1}\right)$ is uniformly bounded for $\tau_{1} \geq 0$ since (21) is asymptotically stable and $|u(t)|$ is also uniformly bounded. The trajectory of $y(t)$ for $t \in\left[\tau_{1}, \tau_{1}+\hat{\tau}_{1}\right]$ satisfies

$$
|y(t)| \leq\left|C \mathrm{e}^{A\left(t-\tau_{1}\right)} x\left(\tau_{1}\right)\right|+\left|\int_{\tau_{1}}^{t} C \mathrm{e}^{A \hat{t}} B u(t-\hat{t}) d \hat{t}\right| .
$$

Moreover, the integral part in (22) satisfies

$$
\begin{aligned}
& \left|\int_{\tau_{1}}^{t} C \mathrm{e}^{A \hat{t}} B u(t-\hat{t}) d \hat{t}\right| \leq\left(\int_{\tau_{1}}^{t}\left(C \mathrm{e}^{A \hat{t}} B\right)^{2} d \hat{t}\right)^{\frac{1}{2}}\left(\int_{\tau_{1}}^{t}\left(u(t-\hat{t})^{2} d \hat{t}\right)^{\frac{1}{2}}\right. \\
& \leq \sqrt{\epsilon}\left(\int_{\tau_{1}}^{t}\left(C \mathrm{e}^{A \hat{t}} B\right)^{2} d \hat{t}\right)^{\frac{1}{2}} \leq \sqrt{\epsilon}\left(\int_{0}^{\infty}\left(C \mathrm{e}^{A \hat{t}} B\right)^{2} d \hat{t}\right)^{\frac{1}{2}} \\
& =\sqrt{\epsilon}\|\hat{G}\|_{2} \leq \delta
\end{aligned}
$$

where $\hat{G}$ is the Laplace transform of $C \mathrm{e}^{A \hat{t}} B$. The first inequality follows from the Cauchy-Swartz inequality. Note also that $\hat{G}$ is strictly proper, due to the structure of (1), (2), with all poles on the open left half plane (from Assumption 11i)), and hence its $\mathcal{H}_{2}$-norm is finite (e.g. [29, Ch. 2]). Hence, noting that $\epsilon$ can be chosen to be arbitrarily small and that for any $\epsilon_{2}$, there exists finite $\hat{t}$ such that $\left|C \mathrm{e}^{A\left(t-\tau_{1}\right)} x\left(\tau_{1}\right)\right| \leq \epsilon_{2}$ for all $t \geq \hat{t}$, it follows that for any $\hat{\epsilon}>0$, there exists $\tilde{\tau}_{1} \in \mathbb{R}_{+}$ such that $|y(t)| \leq \epsilon_{2}+\delta:=\hat{\epsilon}$ for $t \in\left[\tilde{\tau}_{1}, \tau_{1}+\hat{\tau}_{1}\right]$. Finally note that when the value of $\hat{\tau}_{1}$ is sufficiently large, it holds that $\tilde{\tau}_{1}+\bar{\tau}_{1} \leq \tau_{1}+\hat{\tau}_{1}$. The latter completes the proof.

Proof of Theorem 2. The trajectories $z(t, j)$ of system (13) are in general non-unique. However, it can be trivially shown that for each trajectory of $d^{s}(t, j)$, there exists a unique trajectory for $\omega(t, j)$, since $\omega$ is the output of linear system (21) with input $d^{s}$. The analysis below concerns $\omega(t, j)$ given any trajectory $d^{s}(t, j)$ that is compatible with (13) such that the conditions of Theorem 2 hold. For simplicity, in the analysis below we drop the element $j$ from the argument of the solutions, i.e. denoting $x(t, j)$ and $d_{i}^{c}(t, j), i \in N$, by simply $x(t)$ and $d_{i}^{c}(t), i \in N$ respectively.

For system 21, from any initial condition $x(0) \in \mathbb{R}^{n+1}$, $\omega(t)$ is given by

$$
\omega(t)=C \mathrm{e}^{A t} x(0)+\int_{0}^{t} C \mathrm{e}^{A \tau} B u(t-\tau) d \tau,
$$

which suggests that the magnitude of $\omega(t)$ satisfies

$$
|\omega(t)| \leq\left|C \mathrm{e}^{A t} x(0)\right|+\left|\int_{0}^{t} C \mathrm{e}^{A \tau} B u(t-\tau) d \tau\right| .
$$

Since $A$ is Hurwitz, it follows that for any $\hat{\epsilon}>0$ there exists $\tau \in \mathbb{R}_{+}$such that $\left|C \mathrm{e}^{A t} x(0)\right| \leq \hat{\epsilon}$ for all $t \geq \tau$. Furthermore, for the integral part of (24), it holds that $\left|\int_{0}^{t} C \mathrm{e}^{A \tau} B u(t-\tau) d \tau\right| \leq \int_{0}^{t}\left|C \mathrm{e}^{A \tau} B\right| d \tau\|u\|_{\infty} \leq$ $\int_{0}^{\infty}\left|C \mathrm{e}^{A \tau} B\right| d \tau\|u\|_{\infty}=\hat{L}\|u\|_{\infty}$, noting that $\hat{L}$ is bounded from Assumption 11i). Hence, for any $x(0) \in \mathbb{R}^{n+1}$ and any $\epsilon>0$, there exists $\tau \in \mathbb{R}_{+}$such that $|\omega(t)| \leq \epsilon+$ $\hat{L}\left\|d^{s}-d^{s, *}\right\|_{\infty}=\hat{\omega}$ for all $t \geq \tau$.

Now for given $\hat{\omega}$ consider the sets $S(\hat{\omega})=\left\{j: \omega_{j}^{1} \leq\right.$ $\hat{\omega}\}$ and $\hat{S}(\hat{\omega})=N \backslash S(\hat{\omega})$, which should be interpreted as the sets of loads with and without active frequency feedback. In particular, since $|\omega(t)| \leq \hat{\omega}, t \geq \tau$, the dynamics of $d_{j}^{c}$ reduce from (5), (8), to (4), (5), for $j \in \hat{S}(\hat{\omega})$. Furthermore, note that Corollary 1 applies to the set $\hat{S}(\hat{\omega})$, suggesting that $\lim _{|N| \rightarrow \infty} \mathbb{V}\left(d_{\hat{S}(\hat{\omega})}^{s}\right)=0$.

In the arguments below the variables $\tau_{1}, \hat{\tau}_{1}$ and $\tilde{\tau}_{1}, \bar{\tau}_{1}$ are used as in Lemmas 2 and 3 respectively. From Lemma 2, it follows that as $|N| \rightarrow \infty$, then for any $\epsilon_{1}>0, \hat{\tau}_{1} \in \mathbb{R}_{+}$there exists $\tau_{1} \in \mathbb{R}_{+}$such that $\int_{\tau_{1}}^{\tau_{1}+\hat{\tau}_{1}}\left(d_{\hat{S}(\hat{\omega})}^{s}(t)-d_{\hat{S}(\hat{\omega})}^{s, *}\right)^{2} d t \leq \epsilon_{1}$. Note that the value of $\tau_{1}$ depends on $\hat{\tau}_{1}, \epsilon_{1}$ and the initial conditions. It then follows by applying Lemma 3 with $u=$ $\left(d_{\hat{S}(\hat{\omega})}^{s}(t)-d_{\hat{S}(\hat{\omega})}^{s, *}\right)$, that for any $\tilde{\epsilon}>0, \bar{\tau}_{1} \in \mathbb{R}_{+}$, there exists $\tilde{\tau}_{1} \in \mathbb{R}_{+}$such that $|\omega(t)| \leq\left|C \mathrm{e}^{A t} x(0)\right|+\mid \int_{0}^{t} C \mathrm{e}^{A \tau} B u(t-$ $\tau) d \tau|=| C \mathrm{e}^{A t} x(0)|+| \int_{0}^{t} C \mathrm{e}^{A \tau} B\left(d_{\hat{S}(\hat{\omega})}^{s}(t-\tau)-d_{\hat{S}(\hat{\omega})}^{s, *}\right) d \tau \mid+$ $\left|\int_{0}^{t} C \mathrm{e}^{A \tau} B\left(d_{S(\hat{\omega})}^{s}(t-\tau)-d_{S(\hat{\omega})}^{s, *}\right) d \tau\right| \leq \tilde{\epsilon}+\hat{L}\left\|d_{S(\hat{\omega})}^{s}-d_{S(\hat{\omega})}^{s, *}\right\|_{\infty}$ for all $t \in\left[\tilde{\tau}_{1}, \tilde{\tau}_{1}+\bar{\tau}_{1}\right]$. Note that, as follows from the arguments in the proof of Lemma 3 , it holds that $\tilde{\tau}_{1} \geq \tau_{1}$. The rest of the proof is split in two parts, depending on whether $S(\hat{\omega})=\emptyset$ or not.

Part 1: If $S(\hat{\omega})=\emptyset$ then the proof is complete from the above arguments.

Part 2: If $S(\hat{\omega}) \neq \emptyset$, then from Design condition 1 it holds that $\left\|d_{S(\hat{\omega})}^{s}-d_{S(\hat{\omega})}^{s, *}\right\|_{\infty} \leq \sum_{j \in S(\hat{\omega})} \zeta_{j} \bar{d}_{j} \leq \max \left(\hat{L}^{-1}(\hat{\omega}-\right.$ $\delta), 0)$. Then, letting $\bar{\epsilon} \in(0, \delta)$, it follows that $|\omega(t)| \leq \hat{\omega}-$ $(\delta-\bar{\epsilon})=\hat{\omega}_{1}$ for all $t \in\left[\tilde{\tau}_{1}, \tilde{\tau}_{1}+\bar{\tau}_{1}\right]$. Then, note that when $|\omega(t)| \leq \hat{\omega}_{1}$ the set of loads with active frequency feedback reduces to $S\left(\hat{\omega}_{1}\right)$ which satisfies $\left|S\left(\hat{\omega}_{1}\right)\right| \leq|S(\hat{\omega})|$.

The rest of the proof repeats the above argument to construct a decreasing sequence of $\hat{\omega}_{i}$, where the subscript $i$ corresponds to the $i$ th element of the sequence. In particular, since $|\omega(t)| \leq \hat{\omega}_{1}$ for all $t \in\left[\tilde{\tau}_{1}, \tilde{\tau}_{1}+\bar{\tau}_{1}\right]$ it holds that for any $\epsilon_{2}>0, \hat{\tau}_{2} \in \mathbb{R}_{+}$there exists $\tau_{2} \in \mathbb{R}_{+}$such that $\int_{\tau_{2}}^{\tau_{2}+\hat{\tau}_{2}}\left(d_{\hat{S}\left(\hat{\omega}_{1}\right)}^{s}(t)-d_{\hat{S}\left(\hat{\omega}_{1}\right)}^{s, *}\right)^{2} d t \leq \epsilon_{2}$ and hence for any $\bar{\tau}_{2} \in$ $\mathbb{R}_{+}$there exists $\tilde{\tau}_{2} \in \mathbb{R}_{+}$such that $|\omega(t)| \leq \hat{\omega}_{1}-(\delta-\epsilon)=\hat{\omega}_{2}$ for all $t \in\left[\tilde{\tau}_{2}, \tilde{\tau}_{2}+\bar{\tau}_{2}\right]$. The latter follows from Lemma 2 and Lemma 3 as above.

Below, we define $\tau_{i}, \hat{\tau}_{i}$ and $\tilde{\tau}_{i}, \bar{\tau}_{i}$ in analogy to $\tau_{1}, \hat{\tau}_{1}$ and $\tilde{\tau}_{1}, \bar{\tau}_{1}$ corresponding to the $i$ th iteration of the considered sequence. It then follows that the values of $\hat{\tau}_{i}$ and $\bar{\tau}_{i}$ can be selected at each iteration such that $\left[\tilde{\tau}_{i}, \tilde{\tau}_{i}+\bar{\tau}_{i}\right] \subseteq\left[\tau_{i}, \tau_{i}+\hat{\tau}_{i}\right]$ and $\tilde{\tau}_{i+1} \geq \tilde{\tau}_{i}$. Therefore, given that there exists $\hat{\omega}_{i}$ and $\tilde{\tau}_{i}$ such that $|\omega(t)| \leq \hat{\omega}_{i}, t \in\left[\tilde{\tau}_{i}, \tilde{\tau}_{i}+\bar{\tau}_{i}\right]$, where $\bar{\tau}_{i}$ can be 
arbitrarily large, then there exist $\hat{\omega}_{i+1}$ and $\tilde{\tau}_{i+1}>\tilde{\tau}_{i}$ such that $|\omega(t)| \leq \hat{\omega}_{i}-(\delta-\epsilon)=\hat{\omega}_{i+1}$ for all $t \in\left[\tilde{\tau}_{i+1}, \tilde{\tau}_{i+1}+\bar{\tau}_{i+1}\right]$. Note also that $\bar{\tau}_{i}, \bar{\tau}_{i+1}$ can be appropriately selected such that $\left[\tilde{\tau}_{i+1}, \tilde{\tau}_{i+1}+\bar{\tau}_{i+1}\right] \subseteq\left[\tilde{\tau}_{i}, \tilde{\tau}_{i}+\bar{\tau}_{i}\right]$.

Hence, there exists a decreasing sequence of $\hat{\omega}_{i}$ such that $0 \leq \hat{\omega}_{i+1} \leq \hat{\omega}_{i}-(\delta-\bar{\epsilon})$ and $\left|S\left(\hat{\omega}_{i+1}\right)\right| \leq\left|S\left(\hat{\omega}_{i}\right)\right|$. Furthermore, there exists some finite $n$ such that $\hat{\omega}_{n}<\omega_{m}(N)$ which implies that $\left|S\left(\hat{\omega}_{n}\right)\right|=\emptyset$. Then, Lemma 2 holds for the set $N$ and hence it follows that for any $\epsilon>0$, there exists $\tilde{\tau}_{n}$ such that the trajectories of $\omega$ satisfy $|\omega(t)| \leq \epsilon$ for $t \in\left[\tilde{\tau}_{n}, \tilde{\tau}_{n}+\bar{\tau}_{n}\right]$, where $\bar{\tau}_{n}$ can be selected to be arbitrarily large.

\section{REFERENCES}

[1] A. Kasis and I. Lestas, "Frequency regulation with thermostatic load participation in power networks," in 58th IEEE Conference on Decision and Control, 2019.

[2] H. Lund, "Large-scale integration of optimal combinations of pv, wind and wave power into the electricity supply," Renewable energy, vol. 31, no. 4 , pp. 503-515, 2006.

[3] A. Ipakchi and F. Albuyeh, "Grid of the future," IEEE power and energy magazine, vol. 7, no. 2, pp. 52-62, 2009.

[4] A. Molina-Garcia, F. Bouffard, and D. S. Kirschen, "Decentralized demand-side contribution to primary frequency control," IEEE Transactions on Power Systems, 2011.

[5] S. Trip and C. De Persis, "Optimal generation in structure-preserving power networks with second-order turbine-governor dynamics," in Control Conference (ECC), 2016 European, pp. 916-921, IEEE, 2016.

[6] A. Kasis, E. Devane, C. Spanias, and I. Lestas, "Primary frequency regulation with load-side participation-part i: Stability and optimality," IEEE Transactions on Power Systems, vol. 32, no. 5, pp. 3505-3518, 2016.

[7] E. Devane, A. Kasis, M. Antoniou, and I. Lestas, "Primary frequency regulation with load-side participation — part ii: Beyond passivity approaches," IEEE Transactions on Power Systems, vol. 32, no. 5, pp. 3519-3528, 2016.

[8] A. Kasis, E. Devane, and I. Lestas, "Primary frequency regulation in power networks with ancillary service from load-side participation," IFAC-PapersOnLine, vol. 50, no. 1, pp. 4394-4399, 2017.

[9] E. Mallada, C. Zhao, and S. Low, "Optimal load-side control for frequency regulation in smart grids," IEEE Transactions on Automatic Control, vol. 62, no. 12, pp. 6294-6309, 2017.

[10] A. Kasis, N. Monshizadeh, E. Devane, and I. Lestas, "Stability and optimality of distributed secondary frequency control schemes in power networks," IEEE Transactions on Smart Grid, vol. 10, no. 2, pp. $1747-$ 1761, 2017.

[11] A. Kasis, N. Monshizadeh, and I. Lestas, "Secondary frequency control with on-off load side participation in power networks," IEEE Transactions on Control of Network Systems, vol. 7, no. 2, pp. 603-613, 2019.
[12] J.-P. Zimmermann, M. Evans, J. Griggs, N. King, L. Harding, P. Roberts, and C. Evans, "Household electricity survey: A study of domestic electrical product usage," Intertek Testing \& Certification Ltd, 2012.

[13] T. Liu, D. J. Hill, and C. Zhang, "Non-disruptive load-side control for frequency regulation in power systems," IEEE Transactions on Smart Grid, vol. 7, no. 4, pp. 2142-2153, 2016.

[14] A. Kasis, N. Monshizadeh, and I. Lestas, "Primary frequency regulation in power grids with on-off loads: Chattering, limit cycles and convergence to optimality," Automatica, vol. 131, p. 109736, 2021.

[15] J. A. Short, D. G. Infield, and L. L. Freris, "Stabilization of grid frequency through dynamic demand control," IEEE Transactions on power systems, vol. 22, no. 3, pp. 1284-1293, 2007.

[16] D. Angeli and P.-A. Kountouriotis, "A stochastic approach to "dynamicdemand" refrigerator control," IEEE Transactions on control systems technology, vol. 20, no. 3, pp. 581-592, 2012.

[17] M. Aunedi, P.-A. Kountouriotis, J. O. Calderon, D. Angeli, and G. Strbac, "Economic and environmental benefits of dynamic demand in providing frequency regulation," IEEE Transactions on Smart Grid, vol. 4, no. 4, pp. 2036-2048, 2013.

[18] S. H. Tindemans, V. Trovato, and G. Strbac, "Decentralized control of thermostatic loads for flexible demand response," IEEE Transactions on Control Systems Technology, vol. 23, no. 5, pp. 1685-1700, 2015.

[19] L. C. Totu, R. Wisniewski, and J. Leth, "Demand response of a tcl population using switching-rate actuation," IEEE Transactions on Control Systems Technology, vol. 25, no. 5, pp. 1537-1551, 2017.

[20] A. R. Bergen and V. Vittal, Power Systems Analysis. Prentice Hall, 1999.

[21] P. M. Anderson and M. Mirheydar, "A low-order system frequency response model," IEEE Transactions on Power Systems, vol. 5, no. 3, pp. 720-729, 1990.

[22] Q. Shi, F. Li, and H. Cui, "Analytical method to aggregate multi-machine sfr model with applications in power system dynamic studies," IEEE Transactions on Power Systems, vol. 33, no. 6, pp. 6355-6367, 2018.

[23] J. Machowski, J. Bialek, and J. Bumby, Power system dynamics: stability and control. John Wiley \& Sons, 2011.

[24] M. Stadler, W. Krause, M. Sonnenschein, and U. Vogel, "Modelling and evaluation of control schemes for enhancing load shift of electricity demand for cooling devices," Environmental Modelling \& Software, vol. 24, no. 2, pp. 285-295, 2009.

[25] R. Goebel, R. G. Sanfelice, and A. R. Teel, Hybrid Dynamical Systems. modeling, stability, and robustness. Princeton University Press, 2012.

[26] K. Cheung, J. Chow, and G. Rogers, "Power system toolbox, v 3.0," Rensselaer Polytechnic Institute and Cherry Tree Scientific Software, 2009.

[27] K. H. Rosen and K. Krithivasan, Discrete mathematics and its applications: with combinatorics and graph theory. Tata McGraw-Hill Education, 2012.

[28] L. Kuipers and H. Niederreiter, Uniform distribution of sequences. Courier Corporation, 2012

[29] J. C. Doyle, B. A. Francis, and A. R. Tannenbaum, Feedback control theory. Courier Corporation, 2013. 